\title{
Collaborative Systems for Pathology Applications
}

\author{
F. Marcano, N. De Armas, A. Díaz-Cardama and O. Ferrer-Roca*
}

UNESCO Chair of Telemedicine, Department of Pathology, Faculty of Medicine, University of La Laguna, 38071 Tenerife, Canary Islands, Spain

\begin{abstract}
Use of collaborative tools is essential in health applications, especially pathology. This paper presents the results of a huge-size image data collaborative system based on SSVS $\odot$, suitable for telepathology work, which can be implemented in any system working with large images. Quality, image stitching performance in the JPEG200 wavelet domain and image viewing performance were analyzed.
\end{abstract}

Keywords: Collaborative systems, telemedicine, SSVS $^{\circledR}$, JPEG2000, image stitching.

\section{INTRODUCTION}

In telemedicine and distance health applications the use of collaborative systems is essential [1-5]. Employed tools include E-learning/E-coaching [6], online device maintenance [7], image sharing ${ }^{1}$ [9-10], health-grid [2] or even virtual procedures, including not only surgery but also virtual autopsy [10]. These tools are based on multi-agent technology and in a semantic web environment, with telemedicine ontology such as that developed by our group $[1,11]$.

In this paper we present the results of a huge-size image data collaborative system particularly suitable for telepathology, although it may also be applied to any system that employs large images.

Our system is based on Small Size Virtual Slide $\left(\mathrm{SSVS}^{\Theta}\right)^{1}$ images, which are relatively small-size-low-power digital images, capable of supporting a high level of zoom. For collaborative purposes, the images placed in the server are composed of individual fields of view (FOV) of the specimen stitched and compressed in JPEG2000 format due to its advantages on management and $\operatorname{size}^{1}[9,10]$.

The quality, image stitching and viewing performance are analyzed in this paper.

\section{MATERIAL AND METHODS}

The present study used SSVS ${ }^{\oplus}$, described elsewhere, for the analysis of quality and viewing performance.

\section{Image Acquisition}

Image acquisition was performed on a specifically designed software (Texcan-II ${ }^{\circledR}$ [12]) using an AVT Oscar CCD camera of $3272 \times 2469$ pixels, 12-bit color depth, coupled to an Olympus BH-2 with a 4x objective S-Plan N.A. 0.13 and a relay tube NFK $2.5 x$ LD of 125 . Image sharpness was obtained with the zoom focus ${ }^{\odot}$ technique [12].

Captured FOVs were classified in two different sets: I) images with dark corners (vignetting due to differences

\footnotetext{
*Address correspondence to this author at the UNESCO Chair of Telemedicine, Department of Pathology, Faculty of Medicine, University of La Laguna, 38071 Tenerife, Canary Islands, Spain; Tel: +34-922-642015; Fax: +34-922-641855; E-mail: catai@teide.net

'Ferrer-Roca O, Marcano F, Díaz-Cardama A, "Small Size Virtual Slides". Sent to publication. 2007.
}

among CCD size and microscope field projection); II) without vignetting (border correction).

\section{Image Stitching}

Stitching was performed using two commercial products and our own software application.

Commercial products were: Panavue Image Assembler ${ }^{\circledR}$ [13] and Autopano Pro ${ }^{\circledR}$ [14], which provide desirable features for supporting pathology images such as very large file handling (up to $100,000 \times 100,000$ pixels, 16-bits per channel color) and automatic mosaic stitching.

Our own software was built on Kakadu 5.2 library [15]. This application performed image-stitching in the wavelet domain (DWT-Discrete Wavelet transformation) of JPEG2000 images $^{1}[9,15]$.

\section{Collaborative System}

A collaborative system was built using the Kakadu 5.2 [16] software toolkit, integrated by a JPIP-server and a viewer based on JPEG2000 Internet Protocol [17].

\section{Quality Control}

Image quality was evaluated according to presence/absence of artifacts in the stitching process as listed in Table 1.

Table 1. Image Artifacts Detected on SSVS ${ }^{\odot}$

\begin{tabular}{|l|l|l|}
\hline S. No. & Image Artifacts & Description \\
\hline \hline 1 & Dark Spots & $\begin{array}{l}\text { Presence of dark areas due to incorrect image } \\
\text { blending }\end{array}$ \\
\hline 2 & $\begin{array}{l}\text { Inadequate } \\
\text { Image Blending }\end{array}$ & $\begin{array}{l}\text { Image "phantoms" (pixel displacements, } \\
\text { erroneous color register) }\end{array}$ \\
\hline 3 & Misalignments & Partially o totally unmatched images \\
\hline 4 & $\begin{array}{l}\text { Image } \\
\text { Straightening }\end{array}$ & $\begin{array}{l}\text { "wavy" assembled image; image distortion } \\
\text { similar to waves, due to incorrect alignment } \\
\text { of capturing device }\end{array}$ \\
\hline 5 & $\begin{array}{l}\text { Image Distor- } \\
\text { tion }\end{array}$ & $\begin{array}{l}\text { General image distortions, mainly due to } \\
\text { stitching algorithm design or incorrect soft- } \\
\text { ware parameter setup }\end{array}$ \\
\hline
\end{tabular}


Image stitching performance was evaluated, analyzing the processing time plotted against number of image FOVs as shown in Fig. (1). Image viewing performance was also analyzed according the function shown in Fig. (2).

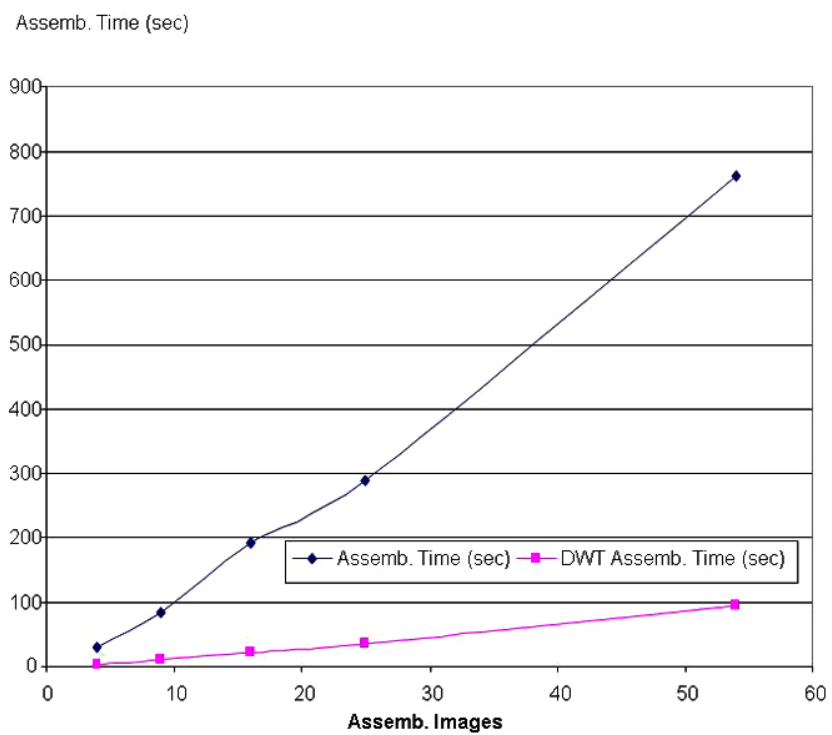

Fig. (1). Stitching performance: a) Using commercial products; b) stitching in the JPEG2000 DWT domain.

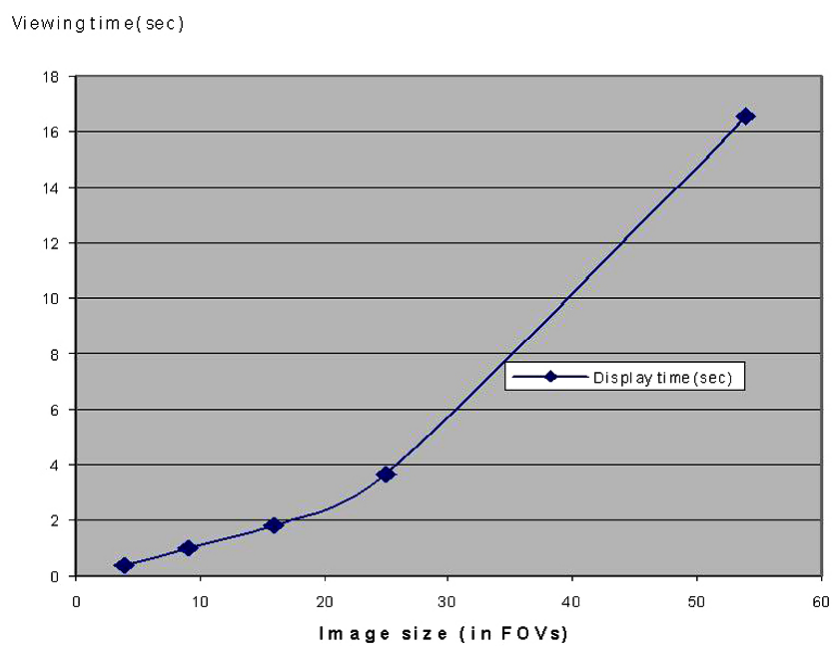

Fig. (2). Image viewing performance.

\section{RESULTS}

The number of images with and without vignetting (set I versus set II) are shown in Tables $\mathbf{2}$ and $\mathbf{3}$.

Table 2. Image Samples for Stitching Experiments $\left(1 \mathrm{~cm}^{2} /\right.$ Each Set)

\begin{tabular}{|l|c|c|}
\hline & Set I & Set II \\
\hline \hline Number of images (by set) & 25 & 36 \\
\hline $\begin{array}{l}\text { Dark/unfocused borders on } \\
\text { each image }\end{array}$ & YES & No \\
\hline Size image component & $3272 \times 2469$ & $2618 \times 1787$ \\
\hline Bit color depth / pixel & 24 & 24 \\
\hline Uncompressed image (KB) & 23,660 & 13,710 \\
\hline
\end{tabular}

The quality control of the image artifacts is shown in Table 4.

Stitching performance is shown in Fig. (1) and viewing performance in Fig. (2). The stitching process in DWT domain runs, on average, more than 8 times faster than naïve uncompress-stitch-compress algorithms, whereas viewing performance in this experiment showed a non-linear tendency on the number of image components.

\section{DISCUSSION}

The use of collaborative environments in the area of health care is gaining ground with the ever-increasing implementation of telemedicine.

Fields of collaboration are image sharing, common diagnosis with images and virtual reality or enhanced imaging. These involve managing huge amounts of large-size images demanding techniques for an efficient handling of storage and transmission such as the ones included in the new DICOM standard versions using JPEG2000.

When images require composition or stitching as is the case with virtual slides in pathology, the problem increase. The present study showed an improvement in storage and distant retrieval of pathology images built with the SSVS ${ }^{\odot}$ technique.

The main performance problem with commercial products is related to image handling, which need to be uncompressed before stitching. We stitched compressed JPEG2000 images directly on the DWT spatial-frequency domain. This approach together with alternatives such as code-block and precinct level stitching has been previously described [15]. As shown in Fig. (1), DWT stitching is more than 8 times faster than stitching with a naïve algorithm (uncompressingstitching-compressing).

Secondly, performance is also influenced by test-bed RAM size, hard disk transfer rates and virtual memory management. In fact RAM access is $10^{3}$ times faster than the fastest hard disks currently available. Greater RAM available implies accelerated stitching process because the operating system does less paging (disk accesses) for appropriate virtual memory management.

However, RAM cannot be expanded in an unlimited way. The 32-bit computer bus architecture only allows up to 4GB RAM, furthermore reduced by installed devices and other operating system features [19]. The 64-bit computer bus architecture provides enough capacity to address RAM/file size beyond $4 \mathrm{~GB}$, thus simplifying whole slide image stitching.

The complete size of a tiled image should not significantly influence transmission performance and image browsing. The reason is that only an area is transmitted over the network. Experiments with different JPIP servers and JPIPcompliant viewers and very large files are recommended to check whether the non-linear tendency shown in Fig. (2) is related to file size or other factors (i.e.: network load, OS virtual memory management or particular JPEG2000-JPIP implementations).

As shown in the results, our system provided adequate and efficient performance, allowing the use of portable or ultra-mobile computers equipped with portable high- 
Table 3. Features of the Assembled Image Sets

\begin{tabular}{|c|c|c|c|}
\hline & Average Size of Uncompressed SSVS (MB) & Average Size of Lossless J2K SSVS (MB) & Compression Factor \\
\hline \hline Set I & 540 & 227 & $2.4: 1$ \\
\hline Set II & 426 & 193 & $2.2: 1$ \\
\hline
\end{tabular}

Table 4. Artifacts Detected

\begin{tabular}{|c|c|c|c|c|c|c|}
\hline Image artifacts & \multicolumn{2}{|c|}{ Panavue ImageAssembler ${ }^{\circledR}$} & \multicolumn{2}{|c|}{ Autopano Pro ${ }^{\circledR}$} & \multicolumn{2}{|c|}{ DWT Stitching } \\
\hline $\begin{array}{c}\text { Inadequate } \\
\text { Blending }\end{array}$ & $\begin{array}{l}\text { Grid-like shadows } \\
\qquad \text { (GLS). } \\
\text { Light differences } \\
\text { among FOVs }\end{array}$ & $\begin{array}{c}\text { GLS } \\
\text { Light difference Phan- } \\
\text { tom borders }\end{array}$ & $\begin{array}{l}\text { Image phantoms } \\
\text { and faded areas. }\end{array}$ & $\begin{array}{l}\text { Image phantoms } \\
\text { and faded areas. }\end{array}$ & Phantom borders & Phantom borders \\
\hline Misalignment & $\begin{array}{c}\text { Empty fields (no } \\
\text { match) }\end{array}$ & $\begin{array}{l}\text { Empty fields (no } \\
\text { match) }\end{array}$ & No & $\begin{array}{c}\text { Empty fields (no } \\
\text { match) }\end{array}$ & No & No \\
\hline Straightening & 1degree image skew. & No skew & No skew. & $\begin{array}{l}\text { Wavy. Missing } \\
\text { areas. }\end{array}$ & No skew & No skew \\
\hline
\end{tabular}

resolution cameras to capture and display the images to be shared [20].

The technique presented here is unique and not reported in the literature that particularly works with virtual slide images of several Gigabytes [21]. Similarly, stitching images in the wavelet domain facilitates computing and increases the speed of building and sharing images as shown in Fig. (1).

In summary: The $\mathrm{SSVS}^{\odot}$ technique with stitching in the DWT domain facilitates storage (due to the small size) and tele-consultation.

\section{REFERENCES}

[1] Ferrer-Roca O. Main Telemedicine applications. In: Ferrer-Roca O, Sosa-Iudicissa M, Eds. Handbook of Telemedicine. Amsterdam: IOS-Press; 1998; 63-97.

[2] BioinfoGRID. Bioinformatics Grid Application for life science. BioinfoGRID Project 2007; [cited 2007 Oct 24th]. Available from http://www.bioinfogrid.eu

[3] HEALTHGRID. Healthgrid 2006; [cited 2007 Oct 24th]. Available from http://www.healthgrid.org

[4] Solomonides T, McClatchey R, Breton V, Legre Y, Nørager S, Eds. From Grid to Healthgrid. Proceedings of Healthgrid 2005. Amsterdam: IOS-Press; 2005.

[5] Pisanelli D, Ed. Ontologies in Medicine. Amsterdam: IOS-Press; 2004.

[6] Adobe Connect. Adobe Systems Inc 2006; [cited 2007 Oct 24th]. Available from http://www.adobe.com/products/connect

[7] Crespo A, Moreu P, Sánchez A. Maintenance Engineering. Madrid:AENOR Editions; 2004.

[8] Marcano F, Ferrer-Roca O, Diaz-Cardama A. Automatic-stitching in pathology. In: Ferrer-Roca O, Ed. CATAI 2007: Telemedicine standardization. Tenerife: CATAI Eds.; 2006; [cited 2007 Oct 24th]; 161-168. Available from: http://www.teide.net/catai/ProceedingsXVWinterCourse/26.htm
[9] Ortiz O, Ruiz V, García I, Marcano F, Ferrer-Roca O. Stitching and remote browsing of images using JPEG2000 and JPIP. In: FerrerRoca O, Ed. CATAI 2007: Telemedicine standardization. Tenerife: CATAI Eds.; 2006; [cited 2007 Oct 24th]; 117-120. Available from: http://www.teide.net/catai/ProceedingsXVWinterCourse/17. htm

[10] Virtopsy®. Virtopsy 2007; [cited 2007 Oct 24th]. Available from: http://www.virtopsy.com

[11] Marcano F, Ferrer-Roca O. Ontologies in e-Health and p-Health In: Ferrer-Roca O, Ed. CATAI 2006: p-Health, Tenerife: CATAI Eds.; 2005; [cited 2007 Oct 24th]; 79-84. Available from: http://www.teide.net/catai/ProceedingXIVWinterCourse/13.htm

[12] Ferrer-Roca O, Marcano F, Diaz-Cardama A. Digital Zooming in Medical Images. In: Ferrer-Roca O, Ed. CATAI-2006: p-health. Tenerife: CATAI Eds.; 2005; [cited 2007 Oct 24th]; 111-118. Available from: http://www.teide.net/catai/ProceedingXIVWinter Course/16.htm

[13] Panavue Image Assembler. Panavue 2006; [cited 2007 Oct 24th] Available from: http://www.panavue.com

[14] Autopano. Autopano 2006; [cited 2007 Oct 24th]. Available from: http://www.autopano.net

[15] Bradley A, Wildermoth M, Mills P. Virtual Microscopy with Extended Depth of Field. In: Proceedings of the Digital Imaging Computing: Techniques and Applications, DICTA 2005. Cairns,Australia:IEEE Computer Society Press; 2005 ;235-242.

[16] Kakadu. Kakadu software 2007; [cited 2007 Oct 24th]. Available from: http://www.kakadusoftware.com

[17] Taubman D, Prandolini R. Architecture. Philosophy and Performance of JPIP: Internet Protocol Standard for JPEG2000. In: Ebrahimi T, Sikora T, Eds. Proceedings of SPIE Vol. 5150, Visual Communications and Image Processing 2003. Santa Clara, CA; 2003; [cited 2007 Oct 24th]; 791-805 Available from: http://www.ee.unsw.edu.au/ taubman/publications_files/JPIParchitecture-vcip03.pdf

[18] Rosenbaum R, Taubman D. Merging Images in JPEG2000Domain. In: Proceedings IASTED-VIIP2003. Benalmadena,Spain; 2003; [cited 2007 Oct 24th]. Available from: http://ww.actapress. com/PDFViewer.aspx ?paperId=14330 
[19] Intel® 945G/945GZ/945P/945PL Express Chipset Family Datasheet. Intel 2005; [cited 2007 Oct 24th]. Available from: http://download.intel.com/design/chipsets/datashts/30750203.pdf

[20] Handheld and Inverted microscopes. Advanced Microscopy Group, Westover Scientific 2007; [cited 2007 Oct 24th]. Available from: http://www.advancedmicroscope.com
[21] Lundin $\mathrm{M}$, Lundin $\mathrm{J}$, Helin H, Isola $\mathrm{J}$. A digital atlas of breast histopathology: an application of web-based virtual microscopy. J Clin Pathol 2004; 57; 1288-91. 